\title{
Under Diagnosis of An Odontogenic Keratocyst- A Controversial Lesion.
}

\author{
Dr. Sherin.A.Khalam*, Dr. Rakesh Koshy Zacariah ${ }^{* *}$ \\ *Sr.Lecturer, **Reader, PMS College Of Dental Science \& Research, Vattappara, Trivandrum, India. \\ Address for correspondence: \\ Dr. Sherin.A.Khalam MSc(Psy), MDS, Division of OMFS PMS College Of Dental Science \& Research, \\ Vattappara, Trivandrum, India.
}

\begin{abstract}
The odontogenic keratocyst usually occurs as a multilocular or unilocular radiolucency often in dentigerous relationship. While the most common site is the posterior portion of the mandible or the mandibular ramus, other locations have been reported such as anterior portion of maxilla and maxillary third molar area . Purpose of this article is to alert the clinician to the fact that odontogenic keratocyst should be included in the differential diagnosis of radiolucencies occurring around an impacted tooth in maxillary anterior region.
\end{abstract}

\section{Introduction}

Odontogenic keratocysts (OKCs) are developmental odontogenic cysts of epithelial origin, first identified and described in 1876 and further characterized by Phillipsen in $1956^{1}$. Pindborg and Hansen suggested the histologic criteria necessary to diagnose OKC in $1962^{2}$.The initial terminology for an odontogenic keratocyst (OKC) was "primordial cyst," as the origin of the lesion was thought to be the tooth primordium. In 1992, the World Health Organization (WHO) histologic typing of odontogenic tumors listed "odontogenic keratocyst" $(\mathrm{OKC})$ as the preferred terminology for such cysts with a keratinized lining ${ }^{3}$.

The odontogenic keratocyst (OKC) is known for its high recurrence rate, aggressive behavior, and its occasional association with the nevoid basal cell carcinoma syndrome (NBCCS) ${ }^{1}$.Three histologic variants were recognized initially: a parakeratinized variant, an orthokeratinized variant, and combination of the two. The less aggressive clinical behavior and recurrence pattern of the orthokeratinized variant ultimately warranted the designation of the orthokeratinized variant as a separate entity, "orthokeratinized odontogenic cyst" (OOC) ${ }^{4}$.

OKC are keratinized epithelium lined cysts of the jaws with well-defined histologic criteria. One clinical feature warranting their recognition and seperation as a distinctive entity is due to their exceedingly high recurrence rate. These lesions may occur anywhere in the jaws but are most commonly seen in mandible rarely in maxilla with males affected more than females.

There are several important things to remember about OKC.

1. Unlike other jaw cysts this cyst has high recurrence rate estimated to be about $30 \%$.

2. Radiographically it is a great mimic. It may resemble periapical cyst, dentigerous cyst, lateral periodontal cyst, nasopalatine duct cyst, traumatic duct cyst and even tumors such as ameloblastoma.

3. A person may have multiple keratocysts at the same time, a little unusual for jaw cysts.

4. If a person has more than one OKC, that person must be investigated for nevoid basal cell carcinoma syndrome.

Philipsen introduced the term OKC in 1956 to describe all odontogenic cysts showing keratinisation of lining epithelium ${ }^{3}$. The OKC represents a specific type of non inflammatory odontogenic cysts originating from primordia, odontogenic epithelium with distinctive microscopic features and potential for recurrence.

Cell rests of dental lamina have been implicated in the pathogenisis of OKC. The dental lamina represents a primordial epithelium having the ability for keratinisation, proliferation and infiltration of connective tissue during odontogenisis, which are the characteristics of both $\mathrm{OKC}$ and ameloblastoma.

The OKC grow primarily in the marrow spaces and in an antero posterior direction. Only when they reach a considerable size do they expand bucco lingually and become evident clinically when seen by the clinician most of them are large and may have perforated the bone especially lingually where the capsule is in close contact with the periosteum ${ }^{3}$.

Malignant transformation of $\mathrm{OKC}$ has been reported ${ }^{4}$, and also changes to ameloblastoma ${ }^{1}$ but has infrequently been reported. OKC may occur in association with basal cell nevus syndrome an autosomal dominant condition characterized by multiple basal cell carcinomas, OKC, skeletal abnormalities.

Recurrence rate reviewed are ranging from 6\%-62\%. It has been proposed that reasons for recurrence include technical and / or surgical difficulties resulting in incomplete cyst removal, the thin and friable nature of capsule, bony perforation, adherence to adjacent soft tissue structures. Patients with multiple OKCs exhibit 
higher rate of recurrence than patients with single OKC. It has been recommended than annual radiographic follow up should be employed indefinitely in the management of these patients. ${ }^{5}$

\section{Case Report.}

A 16 year old girl visited the Dept of Oral And Maxillofacial surgery with a complaint of non painful swelling of right maxillary region. The swelling was reported to be present since one month and was situated in relation to right maxillary canine region, which was clinically missing.

The patient had no significant medical history. On clinical examination, there was a moderate right facial swelling over the right maxillary canine premolar region. The swelling was non- fluctuant on palpation.

The swelling extended into and obliterated the muco buccal fold. A panoramic radiograph revealed a well-circumscribed unilocular radiolucent area that occupied most of left maxilla extending from distal of central incisor to mesial of first permanent molar. The maxillary permanent canine was displaced far superiorly and its eruption was prevented.

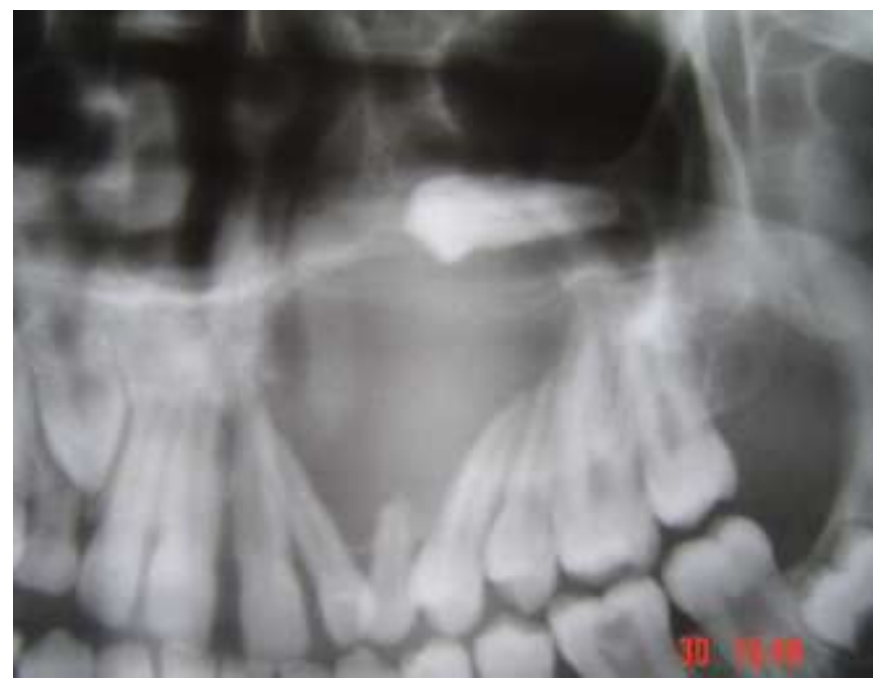

Fig.1. Panoramic radiograph showing the cystic lesion.

The remainder of physical examination was non- contributory. A provisional diagnosis of dentigerous cyst was made on the basis of clinical and radiological features.

We performed an intra oral enucleation through circum gingival incision under GA.

A $2 \times 3 \mathrm{~cm}$ encapsulated cystic lesion was delivered. During enucleation white cheesy material from the cystic mass was collected.

After using copious irrigation, the wound was closed with resorbable sutures. The surgical site healed uneventfully and the patient was rapidly returned to function.

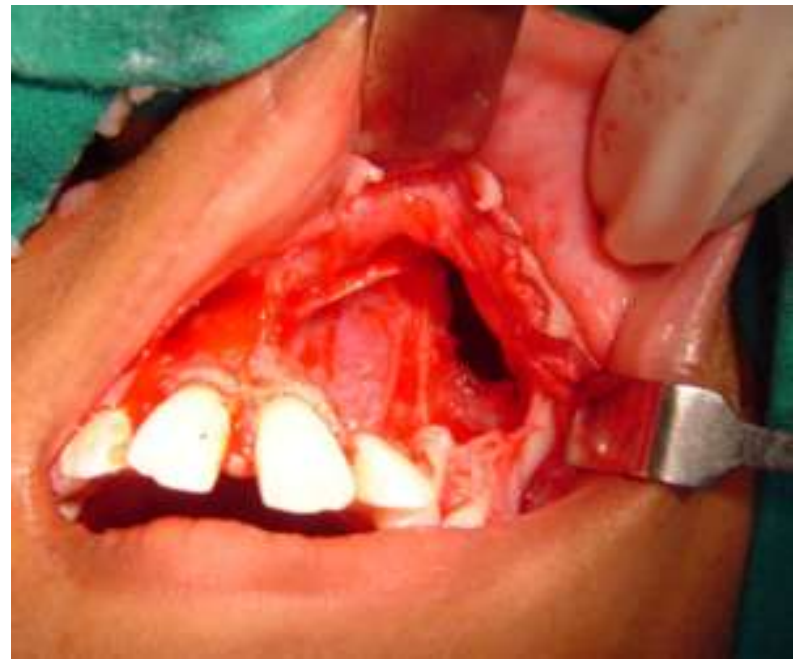

Fig 2. Incison and reflection of mucoperiosteal flap. 


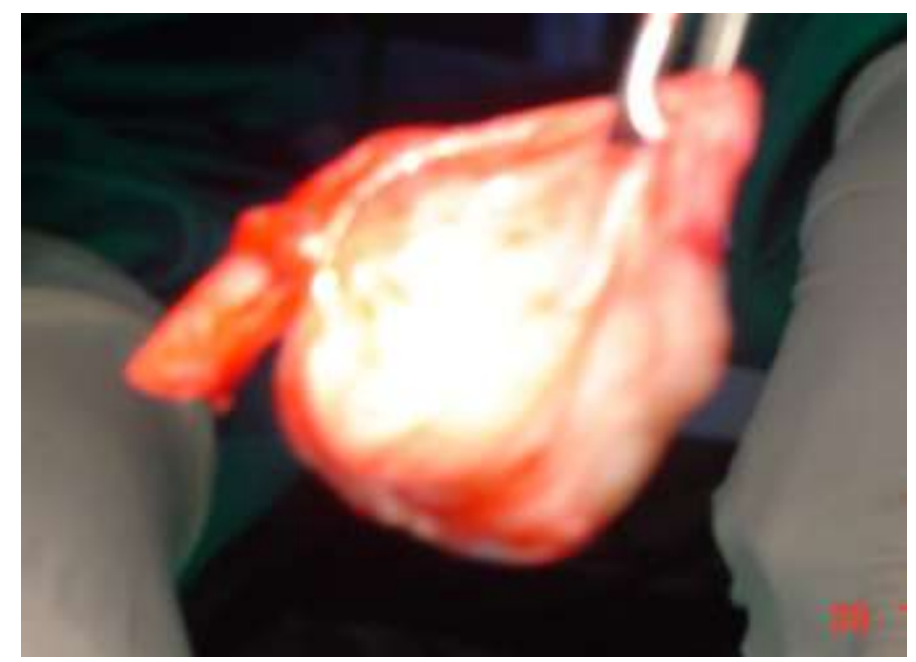

Fig 3. The cystic lesion in toto.

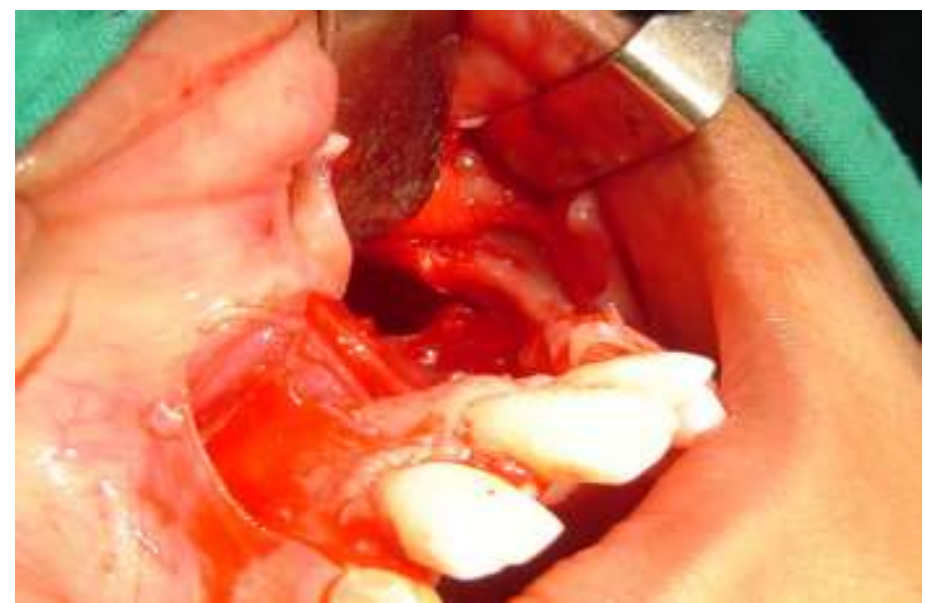

Fig 4. The bony cavity after enucleation

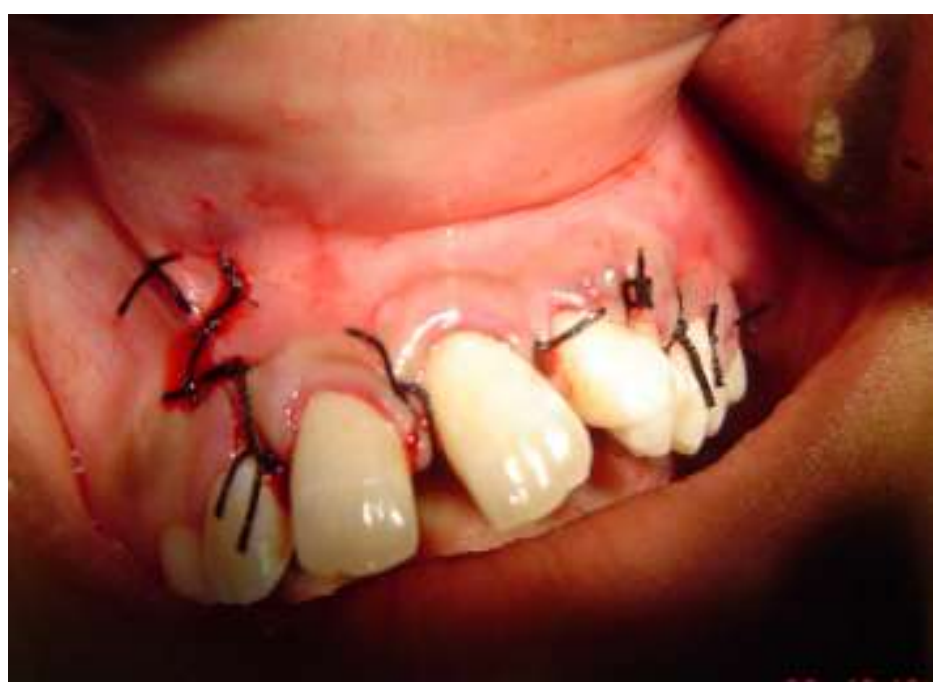

Fig 5. closure.

The specimen was sent for histological examination and the report returned with following findings. 'Microscopically the sections show cystic epithelium which is stratified squamous keratotic with corrugated appearance. The cystic lumen is filled with keratin. There is separation of the epithelium from the connective tissue. The connective tissue capsule is composed of parallely arranged collagen bundles.

Diagnosis of follicular keratocyst with impacted tooth was given. 


\section{Discussion}

The $\mathrm{OKC}$ is a unique clinical and histologic entity with aggressive behaviour. Its frequent recurrence and association with basal cell nevus syndrome warrants care in its diagnosis and management. Most believe the cyst originates in the dental lamina but some support a possible basal cell component origin ${ }^{6}$.

The most common location for keratocyst is mandibular $3^{\text {rd }}$ molar and ramus region. The maxillary $3^{\text {rd }}$ molar is the next most common site followed by mandibular premolar region and maxillary canine region ${ }^{1}$. Brannon found wide variation in provisional clinical diagnosis for cases diagnosed as OKC. ${ }^{1}$ Dentigerous cyst, residual cyst, primordial cyst, lateral periodontal cyst, globulo maxillary cyst were all suspected in some cases. They also present themselves as periapical disease. Wright ${ }^{7}$ emphasizes the importance of including OKC in differential diagnosis of radiolucencies occurring at apices of non-vital teeth. OKCs can also present as eruption cyst and the involved tooth was usually found to erupt through the cyst.

$\mathrm{OKC}$ is often associated with an un-erupted tooth. Browne ${ }^{8}$ has classified the $\mathrm{OKC}$ into follicular and extra follicular types according to its relationship with associated tooth. In this patient we found right permanent canine, crown of which was within the cyst and histology of removed specimen also showed tooth bud in follicular position.

Following differential diagnosis for odontogenic keratocyst have been suggested.

Patients with multiple jaw cysts should always be evaluated for multiple jaw cyst /basal cell nevus syndrome.

Dentigerous cyst

Primordial cyst of the non- OKC type.

Lateral periodontal cyst

Radicular cyst

Residual cyst

Globulomaxillary cyst

Mucous retention cyst

Unicystic or multicystic ameloblastoma.

Numerous surgical modalities have been suggested for treatment of odontogenic keratocyst. These include curettage, enucleation alone, enucleation with cornoy's solutionplaced in the cyst cavity before enucleation or in the bone cavity after enucleation, radical enucleation, marsupialization, and resection.

The type of treatment rendered is controversial, but depends on several factors such as patient's age, location and size of the lesion, and whether the odontogenic keratocyst is primary or recurrent.

Odontogenic keratocyst appears to be a benign disease with a $17-56 \%$ recurrence rate if simple enucleation is used in isolation. If any adjunctive treatment is added, such as application of cornoy's solution, or decompression before enucleation, the recurrence rate is reported to be from $1 \%$ to $8.7 \%$.

Resection is another alternative with a uniform reported recurrence rate of $0 \%$, although extensive nature of surgery might be unacceptable to some clinicians because of benign nature of disease. ${ }^{5}$

\section{Conclusion.}

A case of odontogenic keratocyst mimicking dentigerous cyst radiographically has been presented. The necessity for microscopic examination of all material surgically removed from the jaws has been stressed. We have discussed the clinical implications of a diagnosis of odontogenic keratocyst, that is the high recurrence rate-necessitating close clinical follow up and association of multiple keratocyst with basal cell nevus syndrome.

A long term close follow up is required in order to monitor the possibility of reccurrence of cyst and for the occurrence of basal cell nevus syndrome.

\section{Bibliography}

[1] Sook-Bin Woo,Leon Eisenbud . Odontogenic keratocysts in the anterior maxilla: report of two cases one simulating naso palatine cyst. Oral Surg. Oral Med Oral Pathol 1987:64(4) ;463-5.

[2] Brannon R B.The odontogenic keratocyst. A clinico pathologic study of 312 cases. part I Oral Surg. Oral Med Oral Pathol 1976:42;54-72.

[3] U A Nayak, B.P.Rajesh kumar. A review of odontogenic keratocyst with a report of unusual occurrence in the maxilla. Indian J dent res. 2002:13(3-4); 183-6.

[4] GlenJ Makowski,Stan McGuff. Squamous cell carcinoma in a maxillary odontogenic keratocyst.jour. of oral and maxillo fac surg. 2001; 59:76-80.

[5] Clinical images in oral and maxillofacial radiology. Ouintessence international. $20025(33)$ 400-401.

[6] Brannon R B.The odontogenic keratocyst. A clinico pathologic study of 312 cases. part II Oral Surg. Oral Med Oral Pathol1977; 43; 233-55.

[7] B A Wright G P Wysocki . odontogenic keratocyst presenting as peri apical disease. Oral Surg. Oral Med Oral Pathol 1983 56(4) 425-9

[8] Browne RM . The odontogenic keratocyst. Histologic features and their correlation with clinical behaviour. BRITISH Denatl journ. 1971. 131;248-59. 